\title{
2' Much of a Problem with Hypoglycaemia
}

\author{
N Sukumar ${ }^{1}$, H Venkataraman ${ }^{1}$, J Ayuk ${ }^{1,2}$
}

${ }^{1}$ Department of Endocrinology, University Hospital Birmingham, ${ }^{2}$ University of Birmingham

\section{Case Overview}

- Endocrine referral: 2x hypoglycaemic episodes in non-diabetic patient

- 88 year old man

- Admitted under oncology 4 weeks previously with pulmonary oedema and blocked ureteric stent 11 days after palliative trabecitidine

- Nocturnal hypo-CBG 1.2 when found unrousable from sleep, IV glucose given

- Patient denied any symptoms when questioned

\section{Past medical history}

- Metastatic malignant fibroma of pelvis

- CT (10/16): $16 \mathrm{~cm}$ pelvic mass with small volume lung nodules

- Histology (12/16): solitary fibrous tumour, STAT6 positive, Ki67 30

- For palliative chemotherapy only

- Bilateral hydronephrosis, ureteric stents: 01/17

- Bilateral nephrostomies: 03/17 (for blocked stent and worsening AKI)

- Decompensated heart failure, NYHA Class 3

- Drug history $\rightarrow$ Bisoprolol 2.5mg OD, Fortisip liquid TDS, Hyoscine

butylbromide PRN, Midazolam S/C PRN, Oxynorm IV PRN, Paracetamol 1g QDS

- Social history $\rightarrow$ lives alone, independent ADLs until diagnosis

-Retired machinist

-Non smoker, occasional whiskey

\section{Investigations}

- Initial investigations:-

\begin{tabular}{|l|l|l|}
\hline Test (units) & Result & Normal range \\
\hline Urea (mmol/L) & 22.5 & $3.4-8.0$ \\
\hline Creatinine (umol/L) & 352 & $60-126$ \\
\hline eGFR (ml/min) & 13 & \\
\hline Cortisol (nmol/L) & 412 & $>350$ \\
\hline TSH (mIU/L) & 1.85 & $0.3-4.5$ \\
\hline
\end{tabular}

- Additional blood tests requested during next hypo prior to treatment $\downarrow$ next night

CBG $2.7 \mathrm{mmol} / \mathrm{l}$ - bloods sent off

\begin{tabular}{|l|l|l|}
\hline Test (units) & Result & Normal range \\
\hline Glucose $(\mathrm{mmol} / \mathrm{L})$ & 2.2 & $3.5-11$ \\
\hline Insulin $(\mathrm{pmol} / \mathrm{L})$ & $<10$ & $>20$ \\
\hline C-peptide $(\mathrm{nmol} / \mathrm{L})$ & 380 & \\
\hline IGF-I (nmol/L) & 7.1 & $4.6-23.4$ \\
\hline IGF-II (nmol/L) & 137.2 & \\
\hline IGF-II: IGF-I ratio & 19.3 & $<10$ \\
\hline
\end{tabular}

\section{Management}

- Started Prednisolone 10mg BD $\rightarrow 5 \mathrm{mg}$ BD on discharge

Initial blood glucose chart

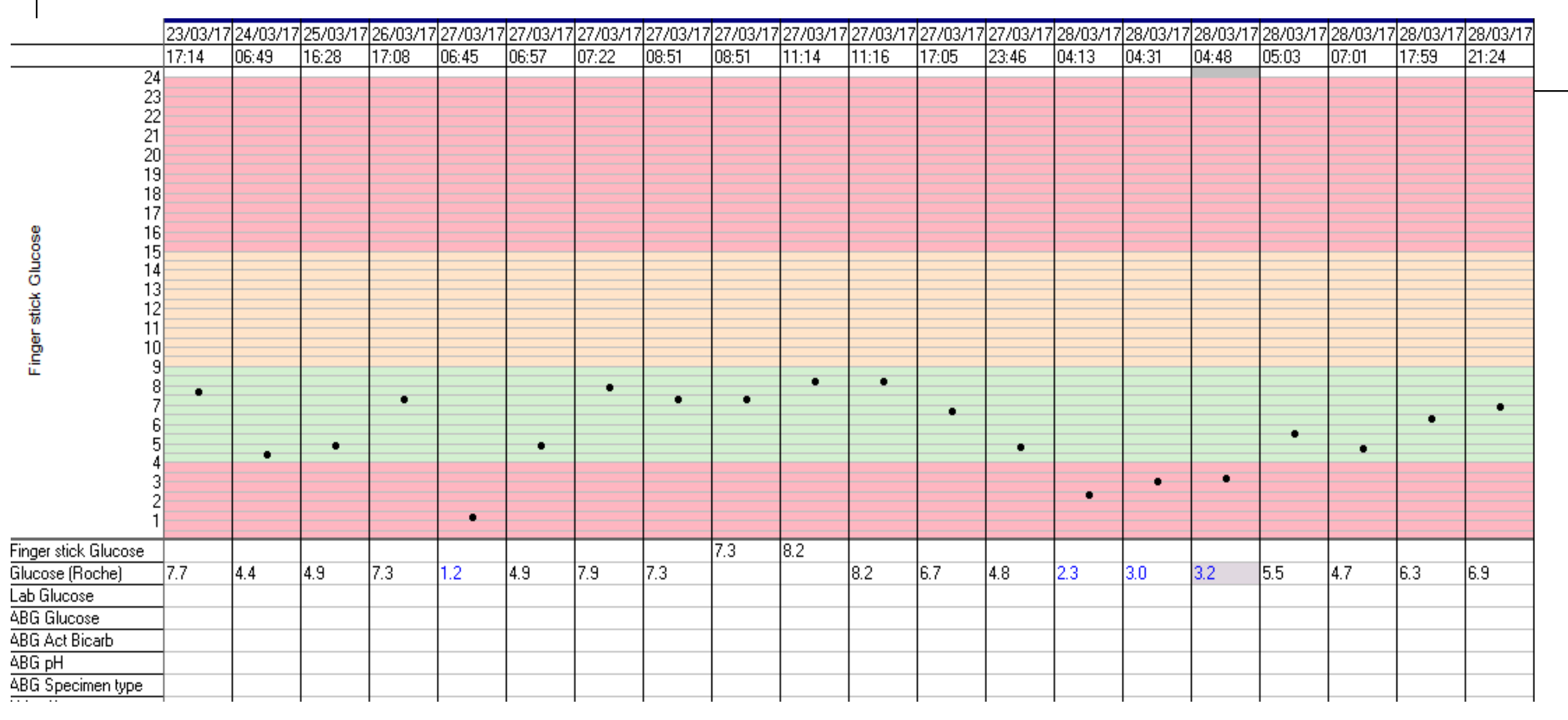

Post-steroid blood glucose chart

\section{Discussion}

\section{Causes of hypoglycaemia}

\section{Insulin mediated}

Drugs

* Exogenous insulin

* Insulin secretagogues

Insulinoma

Functional beta-cell disorders (nesidoblastosis)

* Noninsulinoma pancreatic hypoglycaemia

* Post gastric bypass 'dumping syndrome'

Insulin autoimmune hypoglycaemia

Accidental / surreptitious hypoglycaemia

\section{Non-islet cell tumour hypoglycaemia}

- Complication of certain malignancies resulting in symptomatic severe hypoglycaemia (usually in fasting state)

- $\quad 130$ case reports / small series in English language medical literature in last 30 years ${ }^{1}$

- Occur with $<5 \%$ of solitary fibrous tumours

\section{Pathophysiology}

- Tumours of mesenchymal or epithelial origin ${ }^{1}$

- Solitary fibroma / fibrosarcoma or mesothelioma (22\%)

- Hepatocellular carcinoma (17\%)

- Hermangiopericytoma (7\%)

- Adrenal carcinoma, phaeochromocytoma

- $2 / 3$ retroperitoneal, $1 / 3$ thoracic

- $70 \%$ of tumours $>10 \mathrm{~cm}$ in diameter ${ }^{2}$

- 'Big' IGF-II formed from abnormal processing of pro IGF-II in tumours with aberrant gene transcription / expression

\section{Mechanism of hypoglycaemia}

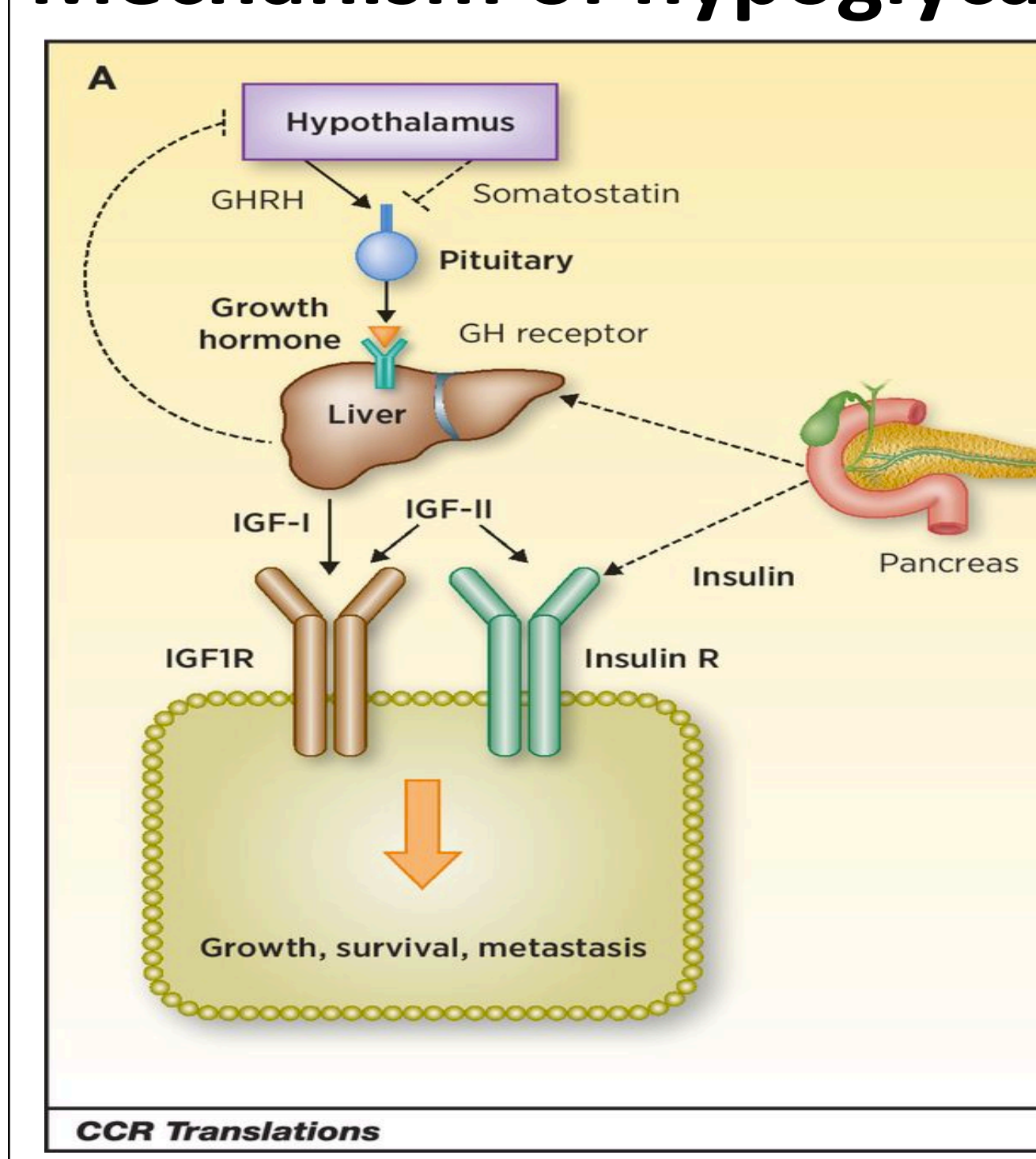

\section{IGF-II production by tumour}

- Acts on insulin receptor to $\uparrow$ glucose utilisation in muscle $+\downarrow$ gluconeogenesis

- Suppresses insulin, glucagon and GH release

- Infiltration of hepatic tissue by tumour

- Destruction of adrenal glands by tumour / haemorrhage

\section{Diagnosis}

- Key feature is $\downarrow \downarrow \downarrow$ glucose/insulin/C-peptide/-hydroxybutarate PLUS $\uparrow$ free IGF-II, IGFII:IGF-I ratio, pro IGF-II levels

Management

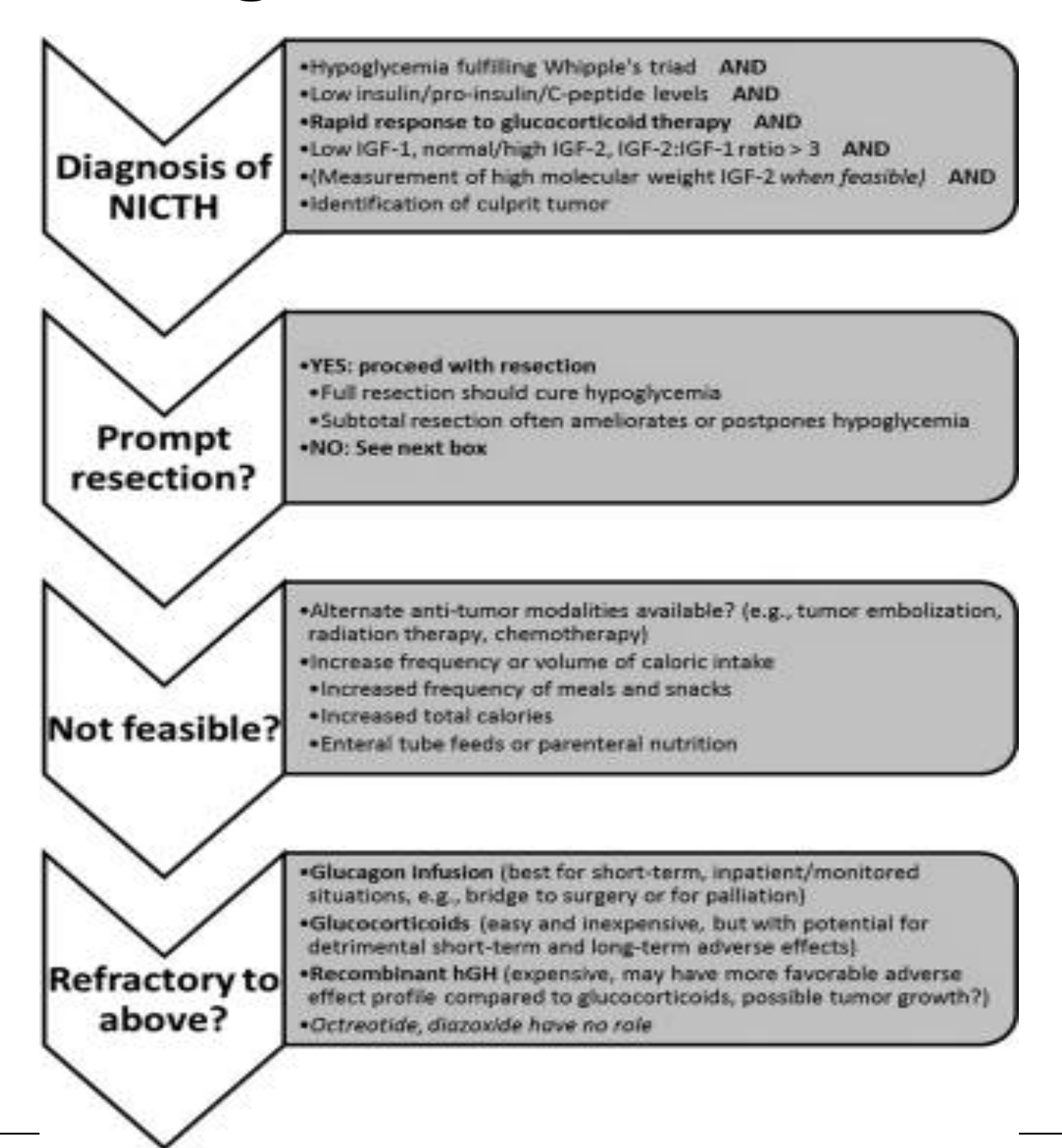

Glucocorticoids

- Suppresses production + increase clearance of IGF-II

- Used in $\sim 25 \%$ of cases

- Typically $30-60 \mathrm{mg} /$ day needed

Recombinant GH

- Caution re: possible effect on tumour growth

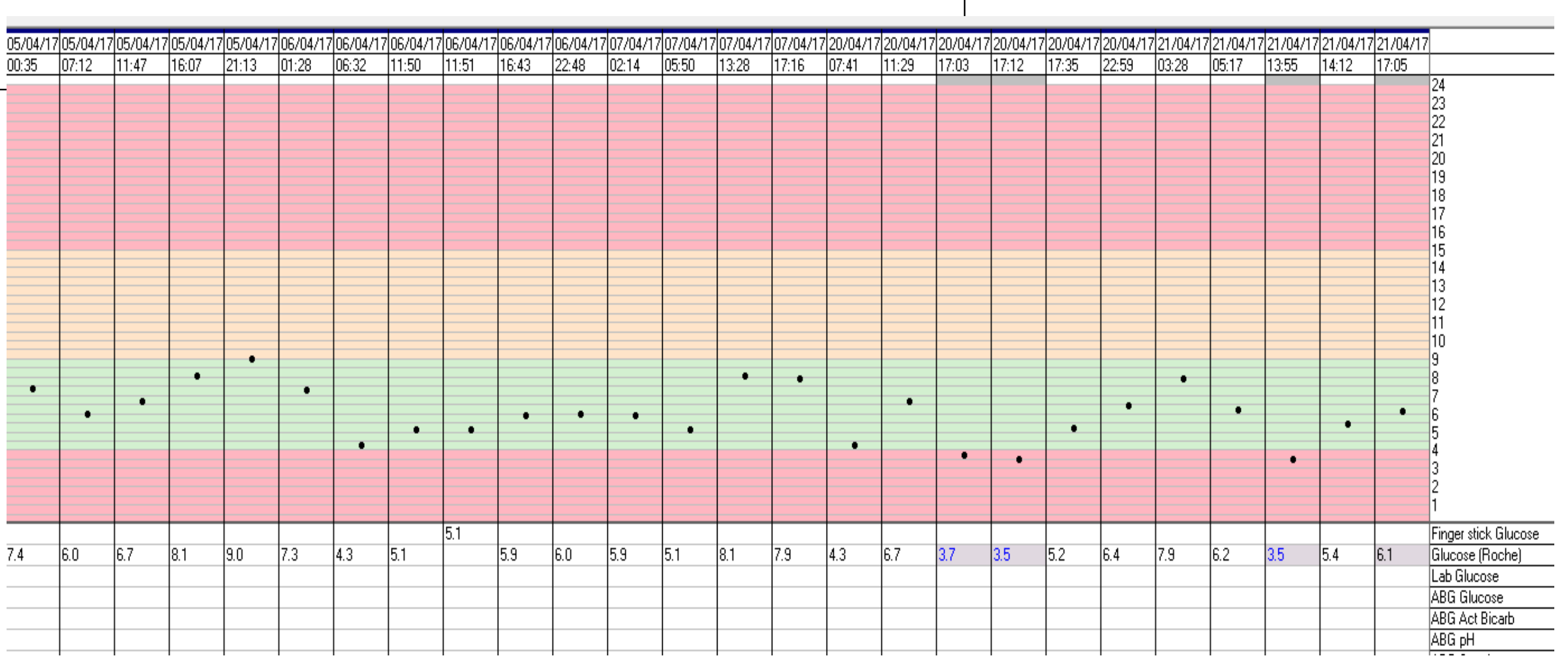

References

1. Bodner TW et al. (2014) Management of non-islet cell tumor hypoglycemia: a clinical review. JCEM; 99(3): 713-22

2. Fukuda I et al. (2006) Clinical features of insulin-like growth factor-II producing non-islet-cell tumour hypoglycemia. Growth Horm IGF Res; 16(4): 211-6 